\title{
PHENOTYPIC DIVERGENCE IN TOMATO GERMPLASM
}

\author{
M.Z. Ullah ${ }^{1}$, L. Hassan ${ }^{2}$, M. Samsuzzaman ${ }^{3}$ and M.A. Main ${ }^{4 *}$ \\ ${ }^{1}$ Bangladesh Institute of Research and Training on Applied Nutrition (BIRTAN) \\ ${ }^{2}$ Department of Genetics \& Plant Breeding, BAU, Mymensingh \\ ${ }^{3}$ Regional Agricultural Rezsearch Station, BARI, Moulvibazar \\ ${ }^{4}$ Tissue Culture Lab, Devine Agro and Tissue Culture Ltd, Jashore.
}

\begin{abstract}
Phenotypic divergence was quantified by multivariate analysis among the 70 genotypes collected from different agro-climatic regions and was available in the gene bank of Energypac Agro Ltd., Gazipur, Bangladesh. Based on the phenotypic value of 11 characters, 70 genotypes were grouped into five clusters. The genotypes of tomato were distributed in different clusters suggesting that no association was found between geographical and phenotypic diversity. Cluster II consisted of maximum twenty three genotypes (32.86\%) followed by cluster III of sixteen genotypes $(22.85 \%)$. Cluster IV and Cluster V comprised of thirteen $(18.57 \%)$ and ten genotypes (14.29\%), respectively. Cluster I consisted of eight genotypes (11.43\%). The highest intra-cluster divergence (0.061) for cluster I was invariably smaller than the lowest inter-cluster divergence between cluster IV and cluster V (2.83), thus authenticating the clustering pattern formed in this study. The intra-cluster divergence ranged from 0.007 to 0.061 , whereas the inter-cluster divergence ranged from 2.83 to 8.34 between clusters IV and V and clusters III and V, respectively. The four characters that played the greatest role in differentiation were locule number per fruit, pericarp thickness, fruits per plant and days to $50 \%$ flowering. Twenty homozygous parents (15 female and five male) were selected from five clusters using range test among genotypes within cluster. From cluster I parents TM409, TM386 and TM528 and from cluster III parents TM403 and TM349 were selected as male. Parents TM356, TM361, TM368, TM371, TM377, TM384, TM422 and TM423 from cluster II; parents TM388, TM390, TM392 and TM410 from cluster IV and parents TM382, TM419 and TM360 from cluster $V$ were selected as female.
\end{abstract}

Keywords: Tomato, Parent selection, Phenotypic value, Multivariate analysis

\footnotetext{
*Corresponding author: zahirsau@gmail.com
} 


\section{INTRODUCTION}

Tomato (Solanum lycopersicum L.) belongs to Solanaceae is one of the important and popular vegetable in the world. It is considered the second important vegetable in Bangladesh after potato because of its wider adaptability, high nutritional value, high yielding potential, multipurpose uses and commercial important (Reddy et al., 2013). Tomato is diploid $(2 \mathrm{n}=24)$ and self-pollinated annual crop. It is source of rich lycopene that acts as an antioxidant which is often colligated with carcinogenesis (Bai and Lindhot, 2007). In Bangladesh the average yield of tomato is very low (13.68 $\mathrm{t} \mathrm{ha}^{-1}$ ) compared to other tropical countries (BBS, 2018).

The knowledge of available genetic diversity is an important factor for any heritable improvement and selecting desirable parents from a germplasm for the successful breeding programe. Among the various methods identified/developed to study the genetic divergence in the genotypes. Mahalanobis $\mathrm{D}^{2}$ (Mahalanobis, 1936) is reliable and the most frequently used. $\mathrm{D}^{2}$ analysis is a useful tool in quantifying the degree of divergence between biological population at genotypic level and to assess relative contribution of different components to the total divergence, both at the inter and intra-cluster levels. The grouping of genotypes into different clusters is done by Tocher's method as described by Rao (1952). An improvement in yield and related traits in self-pollinated crops like tomato is normally achieved by selecting the genotypes with desirable character combinations existing in nature or by hybridization (Reddy et al., 2013; Nalla et al., 2014). It is very useful technique of measuring genetic divergence as reported by various workers (Meena and Bahadur, 2015). Considering the above facts, the present studies had been planned with the objective to assess the extent of phenotypic diversity and identify promising accessions to use in future hybridization program from the available gene pool based on eleven quantitative traits.

\section{MATERIALS AND METHODS}

The present investigation was carried out R\&D center, Energypac Agro Ltd., Gazipur. Seventy tomato genotypes were used as experimental materials (Table 1). Experiment was laid out in RCBD design with three replications during Rabi season of 2012-13. Seed sowing was carried out in October in the nursery. Thirty days old seedlings were transplanted in the field in Mid November with spacing of $40 \mathrm{~cm}$ between plants and $60 \mathrm{~cm}$ between rows. Necessary intercultural operations were carried out during cropping period for proper growth and development of the plants. Data were recorded on various morphological traits such as days to $50 \%$ flowering, flower per cluster, fruits per cluster, fruits per plant, plant height, fruit weight, fruit length, fruit diameter, pericarp thickness, locule number per fruit and fruit yield per plant. The means of characteristics per accessions of three replicates were subjected to $\mathrm{D}^{2}$ and canonical analyzes for genetic divergence (Mahalanobis,1936; Rao, 1952). 
Table 1. List of tomato germplasm and their origin

\begin{tabular}{|c|c|}
\hline Designation & Origin \\
\hline $\begin{array}{l}\text { TM341, TM342, TM343, TM344, TM354, TM355, TM356, TM382, } \\
\text { TM383, TM384, TM388, TM390, TM392, TM393, TM403, TM404, } \\
\text { TM405, TM406, TM407, TM408, TM423, TM425, TM445, TM429, } \\
\text { TM385, TM399, TM491, TM497, TM395, TM360, TM510 }\end{array}$ & India \\
\hline $\begin{array}{l}\text { TM361, TM368, TM369, TM370, TM371, TM374, TM375, TM376, } \\
\text { TM377, TM426, TM428, TM427, TM386, TM357, TM400, TM467, } \\
\text { TM470, TM372, TM462, TM463, TM458, TM460, TM465, TM466, } \\
\text { TM412, TM394, TM349, TM514, TM521, TM524, TM508 }\end{array}$ & Bangladesh \\
\hline TM409, TM528 & China \\
\hline TM515 & Italy \\
\hline TM410, TM417, TM419, TM420, TM422 & Thailand \\
\hline
\end{tabular}

RESULTS

Seventy tomato genotypes were used to study the genetic diversity among them. ANOVA showed the presence of significant variation among the tested genotypes in respect of the yield and yield related characters (Table 2).

\section{PCA analysis}

The results showed that the first principal axis largely accounted for the variation among the genotypes which alone contributed $25.31 \%$ of the total variation (Table 3 ). The first four axis of the principal component with eigen values above unity accounted for $73.59 \%$ of the total variation among the 11 axis. The rest seven axes contributed remaining $26.41 \%$ of total variation.

Table 2. Analysis of variances for yield and yield related characters of 70 genotypes in tomato

\begin{tabular}{|c|c|c|c|c|c|c|c|c|c|c|c|c|}
\hline \multirow{2}{*}{$\begin{array}{c}\text { Variation } \\
\mathrm{s}\end{array}$} & \multirow{2}{*}{ Df } & \multicolumn{11}{|c|}{ MS } \\
\hline & & $\mathrm{D} 50 \% \mathrm{~F}$ & FPC & FRPC & FPP & PH & FW & FL & FD & PT & $\mathrm{LN}$ & FYP \\
\hline Rep & 2 & 14.57 & 0.08 & 2.83 & 23.41 & 35.616 & 593.43 & 0.07 & 0.15 & 0.53 & 0.01 & 0.76 \\
\hline Var & 69 & $\begin{array}{c}162.58 * \\
*\end{array}$ & $\begin{array}{c}2.10^{*} \\
*\end{array}$ & $\begin{array}{c}2.64 * \\
*\end{array}$ & $\begin{array}{c}212.54 * \\
*\end{array}$ & $\begin{array}{c}593.15 * \\
*\end{array}$ & $\begin{array}{c}1437.91 * \\
*\end{array}$ & $\begin{array}{c}1.47 * \\
*\end{array}$ & $\begin{array}{c}0.87 * \\
*\end{array}$ & $\begin{array}{c}2.46^{*} \\
*\end{array}$ & $\begin{array}{c}4.76^{*} \\
*\end{array}$ & $\begin{array}{c}1.30 * \\
*\end{array}$ \\
\hline Error & 138 & 4.63 & 0.52 & 0.42 & 36.24 & 71.44 & 282.81 & 0.15 & 0.14 & 0.55 & 0.46 & 0.42 \\
\hline
\end{tabular}

** $>\mathrm{P} 1 \%, \mathrm{DF}=$ degrees of freedom, $\mathrm{MS}=$ mean sum of square, $\mathrm{D} 50 \% \mathrm{~F}=$ days to $50 \%$ flowering, $\mathrm{FPC}=$ flower per cluster, FRPC $=$ fruits per cluster, $\mathrm{FPP}=$ fruits per cluster, $\mathrm{PH}=$ plant height $(\mathrm{cm}), \mathrm{FW}=$ Fruit weight $(\mathrm{g}), \mathrm{FL}=$ Fruit length $(\mathrm{cm}), \mathrm{FD}=$ Fruit diameter $(\mathrm{cm}), \mathrm{PT}=$ Pericarp thickness $(\mathrm{mm}), \mathrm{LN}=$ Locule number per fruit and $\mathrm{FYP}=$ Fruit yield per plant $(\mathrm{Kg})$. 
Table 3. Eigen values and percent contribution of 11 axis of 70 germplasm

\begin{tabular}{cccc}
\hline Characters & Eigen values & Percent variation & Cumulative \% \\
\hline I & 2.78 & 25.31 & 25.31 \\
II & 2.21 & 20.17 & 45.48 \\
III & 1.65 & 15.01 & 60.49 \\
IV & 1.44 & 13.10 & 73.59 \\
V & 0.94 & 8.55 & 82.14 \\
VI & 0.76 & 6.99 & 89.13 \\
VII & 0.65 & 5.92 & 95.05 \\
VIII & 0.30 & 2.78 & 97.83 \\
IX & 0.14 & 1.34 & 99.17 \\
X & 0.05 & 0.50 & 99.67 \\
XI & 0.03 & 0.33 & 100.00 \\
\hline
\end{tabular}

\section{Clustering and Cluster Distance}

On the basis of nonhierarchical clustering, 70 tomato genotypes were grouped into five clusters (Table 4). Cluster II included of 23 genotypes (32.86\%) and it was followed by cluster III of 16 genotypes (22.85\%), cluster IV comprises 13 genotypes (18.57\%), cluster V consisted of 10 genotypes (14.29\%) while cluster I consisted eight genotypes (11.43\%). The inter-cluster distance ranged from 2.83 to 8.34 (Table $5)$. The inter cluster $D^{2}$ values were maximum (8.34) between the cluster III and cluster V, followed by III and I (6.86) and III and IV (6.32). The intra cluster distance was highest in cluster I (0.061) followed by cluster III (0.016) and the lowest in cluster IV (0.008).

Table 4. Distribution of 70 tomato genotypes in five different clusters

\begin{tabular}{|c|c|c|}
\hline Cluster no. & No. of Genotypes & Genotypes \\
\hline I & 8 & $\begin{array}{l}\text { TM342, TM344, TM370, TM409, TM425, TM386, TM357, } \\
\text { TM528 }\end{array}$ \\
\hline II & 23 & $\begin{array}{l}\text { TM341, TM354, TM355, TM356, TM361, TM368,TM371, } \\
\text { TM376, TM377, TM384, TM405, TM406, TM422, TM423, } \\
\text { TM426, TM427, TM385, TM491, TM463, TM465, TM510, } \\
\text { TM521, TM524 }\end{array}$ \\
\hline III & 16 & $\begin{array}{l}\text { TM343, TM369, TM374, TM403, TM404, TM417, TM420, } \\
\text { TM428, TM462, TM466, TM412, TM394, TM349, TM514, } \\
\text { TM515, TM508 }\end{array}$ \\
\hline IV & 13 & $\begin{array}{l}\text { TM375, TM383, ТM388, TM390, TM392, ТМ393, TM407, } \\
\text { TM408, TM410, TM445, TM497, TM458, TM460 }\end{array}$ \\
\hline $\mathrm{V}$ & 10 & $\begin{array}{l}\text { TM382, TM419, TM429, , TM400, TM399, TM395, TM467, } \\
\text { TM470, TM360, TM372 }\end{array}$ \\
\hline
\end{tabular}


Table 5. Intra (bold) and inter-cluster divergence ( $\mathrm{D}^{2}$ values) among five clusters of tomato

\begin{tabular}{llllll}
\hline Clusters & I & II & III & IV & V \\
\hline I & 0.061 & 5.27 & 6.86 & 4.29 & 6.06 \\
& $(0.246)$ & $(2.30)$ & $(2.62)$ & $(2.07)$ & $(2.46)$ \\
II & & 0.005 & 3.73 & 3.11 & 5.18 \\
& & $(0.070)$ & $(1.93)$ & $(1.76)$ & $(2.28)$ \\
III & & 0.016 & 6.32 & 8.34 \\
& & & $(0.126)$ & $(2.51)$ & $(2.89)$ \\
IV & & & 0.007 & 2.83 \\
& & & & $(0.084)$ & $(1.68)$ \\
V & & & & & 0.011 \\
& & & & & $(0.105)$ \\
\hline
\end{tabular}

$\mathrm{D}$ value in parenthesis

\section{Cluster mean analysis}

Minimum days to $50 \%$ flowering were observed in cluster I (53.92) (Table 6). Maximum (6.47) flowers per cluster were observed in cluster III. Genotypes in cluster III had the highest mean (5.99) for fruits per cluster. Maximum fruits per plant (32.10) and plant height (119.14) were observed in cluster III and cluster I, respectively. Cluster $\mathrm{V}$ and cluster IV had the maximum average fruit weight (123.35) and fruit length (5.99), respectively. Highest fruit diameter (6.29) and pericarp thickness (8.20) both were recorded in cluster V. Minimum locule number per fruit was observed in cluster III (2.80). A highest fruit yield per plant was recorded by the genotype making up cluster I (2.51).

Table 6. Cluster mean values of 11 characters of 70 genotypes

\begin{tabular}{llllll}
\hline Characters & I & II & III & IV & V \\
\hline Day to 50\% flowering & 53.92 & 54.51 & 63.98 & 56.95 & 59.97 \\
Flower per cluster (no.) & 5.81 & 6.15 & 6.47 & 5.85 & 5.99 \\
Fruit per cluster (no.) & 5.88 & 5.24 & 5.99 & 5.05 & 5.59 \\
Fruits per plant (no.) & 28.11 & 25.58 & 32.10 & 19.50 & 17.80 \\
Plant Height (cm) & 119.14 & 82.70 & 87.23 & 89.76 & 80.27 \\
Average fruit weight (g) & 90.81 & 82.94 & 59.88 & 102.45 & 123.35 \\
Fruit length (cm) & 5.61 & 5.47 & 5.27 & 5.99 & 5.97 \\
Fruit diameter (cm) & 5.70 & 5.43 & 4.93 & 5.77 & 6.29 \\
Pericarp thickness (mm) & 7.33 & 7.70 & 7.23 & 7.84 & 8.20 \\
Locule number per fruit & 3.85 & 3.23 & 2.80 & 3.55 & 3.87 \\
Fruit yield per plant (Kg) & 2.51 & 2.05 & 1.92 & 1.99 & 2.16 \\
\hline
\end{tabular}


Table 7. Relative contributions of eleven characters of 70 genotypes

\begin{tabular}{lcc}
\hline \multirow{2}{*}{ Characters } & \multicolumn{2}{c}{ Principal Component } \\
\cline { 2 - 3 } Day to 50\% flowering & Vector-1 & Vector-2 \\
Flower per cluster (no.) & 0.0156 & 0.0262 \\
Fruit per cluster (no.) & -0.1173 & 0.0811 \\
Fruits per plant (no.) & 0.3464 & -0.3603 \\
Plant Height (cm) & 0.0738 & 0.0226 \\
Average fruit weight (g) & -0.0420 & -0.1169 \\
Fruit length (cm) & -0.1248 & 0.0229 \\
Fruit diameter (cm) & 0.1940 & -0.2653 \\
Pericarp thickness (mm) & 0.0546 & -0.8500 \\
Locule number per fruit & 0.0925 & 0.4530 \\
Fruit yield per plant (Kg) & 0.4192 & 0.2609 \\
\hline
\end{tabular}

\section{Selection of parents}

The genotypes of cluster I best in terms of early flowering, high yielder and tall plant (Table 11). The genotypes of cluster II produced early maturing and medium yielder. The genotype of cluster III possessed highest flower and fruit per cluster and highest fruit per plant. The genotypes of cluster IV produced longer fruit and higher fruit weight and the cluster $\mathrm{V}$ possessed highest fruit weight, more fruit diameter, pericarp thickness and locule number per fruit. To select potential homozygous parents from each cluster, DMRT was done within each cluster. That's why mean values with DMRT of studied traits are presented in Table 8-10.

Table 8. Mean performance for 11 traits of genotypes of cluster I \& V

\begin{tabular}{|c|c|c|c|c|c|c|c|c|c|c|c|c|}
\hline Cluster & Genotype & DFPF & FPC (no.) & $\begin{array}{c}\text { FRPC } \\
\text { (no.) }\end{array}$ & $\begin{array}{l}\text { FPP } \\
\text { (no.) }\end{array}$ & $\mathrm{PH}(\mathrm{cm})$ & FW (g) & $\begin{array}{l}\mathrm{FL} \\
(\mathrm{cm})\end{array}$ & $\begin{array}{l}\mathrm{FD} \\
(\mathrm{cm})\end{array}$ & $\begin{array}{c}\mathrm{PT} \\
(\mathrm{mm})\end{array}$ & $\mathrm{LN}$ & $\begin{array}{l}\text { FYP } \\
(\mathrm{Kg})\end{array}$ \\
\hline \multirow{8}{*}{ I } & TM342 & $52.00 \mathrm{~b}$ & $5.67 \mathrm{ab}$ & $5.83-\mathrm{ac}$ & $33.11 \mathrm{a}$ & $111.46 \mathrm{ab}$ & $83.16 b c$ & $5.86 \mathrm{bc}$ & $5.11 \mathrm{~b}$ & $7.72 b$ & $2.33 c$ & $2.76 \mathrm{a}$ \\
\hline & TM344 & $52.67 \mathrm{~b}$ & $6.22 \mathrm{a}$ & $6.67 \mathrm{ab}$ & $29.83 \mathrm{ab}$ & $112.74 \mathrm{ab}$ & $104.86 \mathrm{ab}$ & $7.15 \mathrm{a}$ & $5.50 \mathrm{~b}$ & $8.83 a$ & $2.00 \mathrm{c}$ & $3.13 \mathrm{a}$ \\
\hline & TM370 & $54.00 \mathrm{ab}$ & $6.67 \mathrm{a}$ & $5.56 \mathrm{a}-\mathrm{d}$ & $34.22 \mathrm{a}$ & $137.38 \mathrm{a}$ & $62.52 \mathrm{c}$ & $5.16 \mathrm{~d}-\mathrm{f}$ & $5.19 \mathrm{~b}$ & $6.29 \mathrm{e}$ & $3.42 \mathrm{~b}$ & $2.12 \mathrm{ab}$ \\
\hline & TM409 & $52.33 b$ & $4.78 \mathrm{bc}$ & $5.35 \mathrm{~cd}$ & $14.50 \mathrm{c}$ & $118.17 \mathrm{ab}$ & $93.50 \mathrm{~b}$ & $5.14 \mathrm{ef}$ & $5.75 \mathrm{ab}$ & $7.58 \mathrm{bc}$ & $4.00 \mathrm{ab}$ & $1.38 \mathrm{~b}$ \\
\hline & TM425 & $49.33 c$ & $5.89 \mathrm{ab}$ & $4.44 \mathrm{~d}$ & $34.35 \mathrm{a}$ & $103.07 \mathrm{ab}$ & $84.16 b c$ & $5.52 \mathrm{~cd}$ & $5.51 \mathrm{~b}$ & $7.33 b-d$ & $2.22 \mathrm{c}$ & $2.94 \mathrm{a}$ \\
\hline & TM386 & $52.67 \mathrm{~b}$ & $6.56 \mathrm{a}$ & $5.96 \mathrm{a}-\mathrm{c}$ & $21.50 \mathrm{bc}$ & $87.16 \mathrm{~b}$ & $118.78 \mathrm{a}$ & $5.49 \mathrm{de}$ & $6.38 \mathrm{a}$ & $6.84 \mathrm{c}-\mathrm{e}$ & $4.76 \mathrm{a}$ & $2.53 \mathrm{a}$ \\
\hline & TM357 & $55.67 \mathrm{a}$ & $6.11 \mathrm{a}$ & $6.75 a$ & $24.67 \mathrm{ab}$ & $117.33 \mathrm{ab}$ & $94.85 \mathrm{~b}$ & $5.03 \mathrm{f}$ & $5.81 \mathrm{ab}$ & $6.75 \mathrm{de}$ & $4.17 \mathrm{ab}$ & $2.36 \mathrm{ab}$ \\
\hline & TM528 & $48.00 \mathrm{c}$ & $3.78 \mathrm{c}$ & $5.44 b-d$ & $29.63 \mathrm{ab}$ & $115.94 \mathrm{ab}$ & $84.39 b c$ & $5.99 \mathrm{~b}$ & $5.73 \mathrm{ab}$ & $8.13 \mathrm{ab}$ & $3.67 \mathrm{ab}$ & $2.49 \mathrm{ab}$ \\
\hline V & TM382 & $57.00 \mathrm{~d}$ & $5.11 \mathrm{c}$ & $4.67 b c$ & $22.67 \mathrm{a}-\mathrm{c}$ & $73.73 \mathrm{c}$ & $112.53 \mathrm{a}$ & $6.17 \mathrm{~b}$ & $6.17 b c$ & $7.95 \mathrm{a}$ & $4.67 \mathrm{~b}$ & $2.58 \mathrm{a}$ \\
\hline
\end{tabular}




\begin{tabular}{ccccccccccccc}
\hline Cluster & Genotype & DFPF & FPC (no.) & $\begin{array}{c}\text { FRPC } \\
(\text { no. })\end{array}$ & $\begin{array}{c}\text { FPP } \\
(\text { no. })\end{array}$ & PH (cm) & FW (g) & $\begin{array}{c}\text { FL } \\
(\mathrm{cm})\end{array}$ & $\begin{array}{c}\text { FD } \\
(\mathrm{cm})\end{array}$ & $\begin{array}{c}\text { PT } \\
(\mathrm{mm})\end{array}$ & $\begin{array}{c}\text { LN } \\
\text { FYP } \\
(\mathrm{Kg})\end{array}$ \\
\hline & TM419 & $67.33 \mathrm{~b}$ & $7.33 \mathrm{a}$ & $7.00 \mathrm{a}$ & $24.53 \mathrm{a}$ & $137.00 \mathrm{a}$ & $119.07 \mathrm{a}$ & $5.00 \mathrm{c}$ & $7.03 \mathrm{a}$ & $5.99 \mathrm{~b}$ & $9.00 \mathrm{a}$ & $2.90 \mathrm{a}$ \\
& TM429 & $75.33 \mathrm{a}$ & $5.22 \mathrm{c}$ & $5.35 \mathrm{bc}$ & $15.50 \mathrm{c}-\mathrm{e}$ & $79.17 \mathrm{bc}$ & $115.28 \mathrm{a}$ & $7.08 \mathrm{a}$ & $5.68 \mathrm{c}$ & $9.00 \mathrm{a}$ & $2.00 \mathrm{c}$ & $1.70 \mathrm{a}$ \\
& TM400 & $54.67 \mathrm{~d}$ & $6.78 \mathrm{ab}$ & $7.00 \mathrm{a}$ & $15.82 \mathrm{~b}-\mathrm{e}$ & $83.00 \mathrm{bc}$ & $124.04 \mathrm{a}$ & $5.92 \mathrm{bc}$ & $6.17 \mathrm{bc}$ & $8.14 \mathrm{a}$ & $4.37 \mathrm{~b}$ & $1.96 \mathrm{a}$ \\
& TM399 & $56.67 \mathrm{~d}$ & $6.00 \mathrm{bc}$ & $6.67 \mathrm{a}$ & $21.83 \mathrm{a}-\mathrm{c}$ & $87.00 \mathrm{~b}$ & $122.50 \mathrm{a}$ & $5.51 \mathrm{bc}$ & $6.33 \mathrm{a}-\mathrm{c}$ & $8.08 \mathrm{a}$ & $4.00 \mathrm{bc}$ & $2.70 \mathrm{a}$ \\
& TM395 & $60.33 \mathrm{c}$ & $6.00 \mathrm{bc}$ & $4.22 \mathrm{c}$ & $15.11 \mathrm{c}-\mathrm{e}$ & $74.67 \mathrm{c}$ & $130.20 \mathrm{a}$ & $5.87 \mathrm{bc}$ & $6.33 \mathrm{a}-\mathrm{c}$ & $7.92 \mathrm{a}$ & $3.17 \mathrm{bc}$ & $1.99 \mathrm{a}$ \\
TM467 & $62.00 \mathrm{c}$ & $6.22 \mathrm{a}-\mathrm{c}$ & $5.44 \mathrm{~b}$ & $9.67 \mathrm{e}$ & $77.67 \mathrm{bc}$ & $133.68 \mathrm{a}$ & $5.87 \mathrm{bc}$ & $6.42 \mathrm{a}-\mathrm{c}$ & $8.17 \mathrm{a}$ & $4.17 \mathrm{bc}$ & $1.26 \mathrm{a}$ \\
TM470 & $62.67 \mathrm{c}$ & $6.22 \mathrm{a}-\mathrm{c}$ & $4.67 \mathrm{bc}$ & $18.00 \mathrm{a}-\mathrm{d}$ & $81.00 \mathrm{bc}$ & $123.08 \mathrm{a}$ & $6.18 \mathrm{~b}$ & $6.93 \mathrm{ab}$ & $9.28 \mathrm{a}$ & $4.11 \mathrm{bc}$ & $2.22 \mathrm{a}$ \\
TM360 & $61.33 \mathrm{c}$ & $6.33 \mathrm{a}-\mathrm{c}$ & $7.11 \mathrm{a}$ & $24.00 \mathrm{ab}$ & $80.00 \mathrm{bc}$ & $113.86 \mathrm{a}$ & $5.95 \mathrm{bc}$ & $5.92 \mathrm{c}$ & $8.17 \mathrm{a}$ & $2.83 \mathrm{bc}$ & $2.77 \mathrm{a}$ \\
TM372 & $61.00 \mathrm{c}$ & $5.78 \mathrm{bc}$ & $5.35 \mathrm{bc}$ & $11.50 \mathrm{de}$ & $73.83 \mathrm{c}$ & $143.55 \mathrm{a}$ & $5.94 \mathrm{bc}$ & $6.74 \mathrm{ab}$ & $9.17 \mathrm{a}$ & $3.83 \mathrm{bc}$ & $1.65 \mathrm{a}$ \\
\hline
\end{tabular}

Table 9. Mean performance for 11 traits of genotypes of cluster II

\begin{tabular}{|c|c|c|c|c|c|c|c|c|c|c|c|c|}
\hline Cluster & Genotype & DFPF & FPC (no.) & FRPC (no.) & FPP (no.) & $\mathrm{PH}(\mathrm{cm})$ & FW (g) & FL $(\mathrm{cm})$ & $\mathrm{FD}(\mathrm{cm})$ & PT (mm) & $\mathrm{LN}$ & FYP $(\mathrm{Kg})$ \\
\hline \multirow{23}{*}{ II } & TM341 & $55.00 \mathrm{~d}-\mathrm{f}$ & $8.11 \mathrm{a}$ & $6.33 \mathrm{a}-\mathrm{d}$ & $25.33 b-d$ & $78.01 \mathrm{c}-\mathrm{f}$ & $76.08 \mathrm{a}-\mathrm{c}$ & $5.97 \mathrm{a}-\mathrm{c}$ & $5.00 \mathrm{de}$ & 7.75a-f & $2.33 \mathrm{gh}$ & $1.94 \mathrm{a}-\mathrm{e}$ \\
\hline & TM354 & $48.33 \mathrm{~g}-\mathrm{j}$ & $6.74 \mathrm{~b}$ & $4.44 \mathrm{~g}$ & $20.67 b-d$ & $79.53 b-f$ & $85.98 \mathrm{a}-\mathrm{c}$ & $5.20 \mathrm{c}-\mathrm{f}$ & $5.75 \mathrm{a}-\mathrm{c}$ & $7.97 \mathrm{a}-\mathrm{e}$ & $3.50 \mathrm{c}-\mathrm{f}$ & $1.84 \mathrm{a}-\mathrm{e}$ \\
\hline & TM355 & $61.33 \mathrm{bc}$ & $5.89 \mathrm{~b}-\mathrm{d}$ & $4.22 \mathrm{~g}$ & $16.67 \mathrm{~cd}$ & $84.31 \mathrm{a}-\mathrm{e}$ & $74.96 \mathrm{bc}$ & $5.80 \mathrm{a}-\mathrm{e}$ & $4.88 \mathrm{e}$ & $7.56 \mathrm{~b}-\mathrm{g}$ & $2.33 \mathrm{gh}$ & $1.25 \mathrm{de}$ \\
\hline & TM356 & $51.67 \mathrm{e}-\mathrm{i}$ & $6.44 \mathrm{~b}$ & $5.44 \mathrm{c}-\mathrm{g}$ & $28.08 \mathrm{a}-\mathrm{c}$ & 83.86a-e & $91.99 \mathrm{ab}$ & $5.75 \mathrm{~b}-\mathrm{e}$ & $5.77 \mathrm{a}-\mathrm{c}$ & $7.30 \mathrm{c}-\mathrm{g}$ & $4.00 \mathrm{~b}-\mathrm{d}$ & $2.58 \mathrm{a}-\mathrm{c}$ \\
\hline & TM361 & $53.67 \mathrm{e}-\mathrm{g}$ & $4.89 \mathrm{~cd}$ & $4.67 \mathrm{fg}$ & $23.67 \mathrm{~b}-\mathrm{d}$ & 87.13a-d & $84.38 \mathrm{a}-\mathrm{c}$ & $5.32 \mathrm{c}-\mathrm{f}$ & $5.68 \mathrm{a}-\mathrm{d}$ & $7.00 \mathrm{~d}-\mathrm{g}$ & $4.67 \mathrm{~b}$ & $2.04 \mathrm{a}-\mathrm{e}$ \\
\hline & TM368 & $53.00 \mathrm{e}-\mathrm{h}$ & $6.56 \mathrm{~b}$ & $7.11 \mathrm{a}$ & $33.58 \mathrm{ab}$ & $79.74 b-f$ & $68.34 \mathrm{c}$ & $5.09 \mathrm{ef}$ & $5.25 \mathrm{~b}-\mathrm{e}$ & $6.30 \mathrm{~g}$ & $3.27 \mathrm{c}-\mathrm{g}$ & $2.29 \mathrm{a}-\mathrm{e}$ \\
\hline & TM371 & $47.33 \mathrm{~h}-\mathrm{j}$ & $6.78 b$ & $5.78 \mathrm{~b}-\mathrm{f}$ & $33.22 \mathrm{ab}$ & $78.66 \mathrm{~b}-\mathrm{f}$ & $86.88 \mathrm{a}-\mathrm{c}$ & $5.23 \mathrm{c}-\mathrm{f}$ & $5.72 \mathrm{a}-\mathrm{c}$ & $8.90 \mathrm{a}$ & $3.27 \mathrm{c}-\mathrm{g}$ & $2.87 \mathrm{a}$ \\
\hline & TM376 & $57.00 \mathrm{c}-\mathrm{e}$ & $6.33 b$ & $6.22 \mathrm{a}-\mathrm{e}$ & $20.67 b-d$ & $68.97 f$ & $95.32 \mathrm{a}$ & $5.33 \mathrm{c}-\mathrm{f}$ & $5.97 \mathrm{ab}$ & $7.67 \mathrm{a}-\mathrm{f}$ & $4.67 \mathrm{~b}$ & $1.98 \mathrm{a}-\mathrm{e}$ \\
\hline & TM377 & $64.33 \mathrm{~b}$ & $5.78 \mathrm{~b}-\mathrm{d}$ & $6.75 \mathrm{ab}$ & $15.33 \mathrm{~cd}$ & 73.33ef & $92.07 \mathrm{ab}$ & $5.57 \mathrm{~b}-\mathrm{f}$ & $5.37 \mathrm{a}-\mathrm{e}$ & $6.56 \mathrm{fg}$ & $3.78 \mathrm{~b}-\mathrm{e}$ & $1.38 \mathrm{c}-\mathrm{e}$ \\
\hline & TM384 & $50.33 \mathrm{f}-\mathrm{j}$ & $6.44 \mathrm{~b}$ & $4.44 \mathrm{~g}$ & $21.60 b-d$ & $74.81 \mathrm{~d}-\mathrm{f}$ & $84.93 \mathrm{a}-\mathrm{c}$ & $5.49 \mathrm{c}-\mathrm{f}$ & 5.56a-e & $8.19 \mathrm{a}-\mathrm{d}$ & $2.71 \mathrm{e}-\mathrm{h}$ & $1.83 \mathrm{a}-\mathrm{e}$ \\
\hline & TM405 & $55.33 \mathrm{~d}-\mathrm{f}$ & $6.67 \mathrm{~b}$ & $5.00 \mathrm{e}-\mathrm{g}$ & $20.34 b-d$ & $90.13 a-c$ & $86.12 a-c$ & $5.33 \mathrm{c}-\mathrm{f}$ & $5.33 \mathrm{a}-\mathrm{e}$ & $8.38 \mathrm{a}-\mathrm{c}$ & $2.00 \mathrm{~h}$ & $1.45 \mathrm{c}-\mathrm{e}$ \\
\hline & TM406 & $46.00 \mathrm{ij}$ & $5.78 \mathrm{~b}-\mathrm{d}$ & $5.00 \mathrm{e}-\mathrm{g}$ & $15.78 \mathrm{~cd}$ & $77.82 \mathrm{c}-\mathrm{f}$ & $87.30 \mathrm{a}-\mathrm{c}$ & $5.19 \mathrm{c}-\mathrm{f}$ & $5.50 \mathrm{a}-\mathrm{e}$ & $6.79 \mathrm{e}-\mathrm{g}$ & $4.33 b c$ & $1.38 \mathrm{c}-\mathrm{e}$ \\
\hline & TM422 & $50.00 \mathrm{f}-\mathrm{j}$ & $6.11 \mathrm{bc}$ & $4.44 \mathrm{~g}$ & $22.73 b-d$ & $92.32 \mathrm{ab}$ & $70.83 c$ & $5.93 \mathrm{a}-\mathrm{d}$ & $5.27 \mathrm{~b}-\mathrm{e}$ & $8.17 \mathrm{a}-\mathrm{d}$ & $2.33 \mathrm{gh}$ & $1.61 \mathrm{~b}-\mathrm{e}$ \\
\hline & TM423 & $50.33 \mathrm{f}-\mathrm{j}$ & $4.67 \mathrm{~d}$ & $6.67 \mathrm{a}-\mathrm{c}$ & $33.50 \mathrm{ab}$ & $88.42 \mathrm{a}-\mathrm{d}$ & $82.28 \mathrm{a}-\mathrm{c}$ & $5.38 \mathrm{c}-\mathrm{f}$ & $5.52 \mathrm{a}-\mathrm{e}$ & $7.83 \mathrm{a}-\mathrm{f}$ & $3.00 \mathrm{~d}-\mathrm{h}$ & $2.76 \mathrm{ab}$ \\
\hline & TM426 & $52.33 \mathrm{e}-\mathrm{h}$ & $4.89 \mathrm{~cd}$ & $4.22 \mathrm{~g}$ & $38.44 a$ & $78.66 c-f$ & $75.65 \mathrm{a}-\mathrm{c}$ & $5.31 \mathrm{c}-\mathrm{f}$ & $5.12 \mathrm{c}-\mathrm{e}$ & 8.00a-e & $2.33 \mathrm{gh}$ & $2.90 \mathrm{a}$ \\
\hline & TM427 & $51.33 \mathrm{ei}$ & $6.67 \mathrm{~b}$ & $4.67 \mathrm{fg}$ & $32.48 \mathrm{ab}$ & $84.33 \mathrm{a}-\mathrm{e}$ & $72.04 \mathrm{bc}$ & $5.18 \mathrm{~d}-\mathrm{f}$ & $5.27 \mathrm{~b}-\mathrm{e}$ & $7.09 \mathrm{c}-\mathrm{g}$ & $3.33 \mathrm{c}-\mathrm{g}$ & $2.34 \mathrm{a}-\mathrm{d}$ \\
\hline & TM385 & $56.67 \mathrm{c}-\mathrm{e}$ & $6.56 \mathrm{~b}$ & $5.35 \mathrm{~d}-\mathrm{g}$ & $25.89 \mathrm{a}-\mathrm{d}$ & $89.78 \mathrm{a}-\mathrm{c}$ & $84.68 \mathrm{a}-\mathrm{c}$ & $5.51 \mathrm{c}-\mathrm{f}$ & $5.48 \mathrm{a}-\mathrm{e}$ & $8.39 \mathrm{a}-\mathrm{c}$ & $2.55 \mathrm{f}-\mathrm{h}$ & $2.20 \mathrm{a}-\mathrm{e}$ \\
\hline & TM491 & $45.33 \mathrm{j}$ & 5.56b-d & $4.44 \mathrm{~g}$ & $12.77 \mathrm{~d}$ & $79.40 \mathrm{~b}-\mathrm{e}$ & $82.49 \mathrm{a}-\mathrm{c}$ & $4.92 \mathrm{f}$ & $5.56 \mathrm{a}-\mathrm{e}$ & $5.19 \mathrm{~h}$ & $6.87 \mathrm{a}$ & $1.05 \mathrm{e}$ \\
\hline & TM463 & $71.00 \mathrm{a}$ & $6.22 b c$ & $5.78 \mathrm{~b}-\mathrm{f}$ & $26.00 \mathrm{a}-\mathrm{d}$ & $87.33 \mathrm{a}-\mathrm{d}$ & $81.37 \mathrm{a}-\mathrm{c}$ & $6.48 \mathrm{a}$ & $4.89 \mathrm{e}$ & $8.89 \mathrm{a}$ & $2.00 \mathrm{~h}$ & $2.11 \mathrm{a}-\mathrm{e}$ \\
\hline & TM465 & $59.33 \mathrm{~b}-\mathrm{d}$ & $6.22 b c$ & $5.96 a-e$ & $23.00 \mathrm{~b}-\mathrm{d}$ & $95.67 \mathrm{a}$ & $75.12 \mathrm{a}-\mathrm{c}$ & $4.86 \mathrm{f}$ & $5.26 \mathrm{~b}-\mathrm{e}$ & $7.50 \mathrm{~b}-\mathrm{g}$ & $2.58 \mathrm{f}-\mathrm{h}$ & $1.74 \mathrm{a}-\mathrm{e}$ \\
\hline & TM510 & $60.33 \mathrm{~b}-\mathrm{d}$ & $5.44 \mathrm{~b}-\mathrm{d}$ & $7.00 \mathrm{ab}$ & $21.00 \mathrm{~b}-\mathrm{d}$ & 82.21a-f & $84.83 \mathrm{a}-\mathrm{c}$ & $6.29 \mathrm{ab}$ & $5.13 \mathrm{c}-\mathrm{e}$ & $8.67 \mathrm{ab}$ & $2.00 \mathrm{~h}$ & $1.81 \mathrm{a}-\mathrm{e}$ \\
\hline & TM521 & $56.33 \mathrm{c}-\mathrm{e}$ & $6.14 b c$ & $4.44 \mathrm{~g}$ & $33.00 \mathrm{ab}$ & 84.06a-e & $76.80 \mathrm{a}-\mathrm{c}$ & $5.57 \mathrm{~b}-\mathrm{f}$ & $5.17 \mathrm{c}-\mathrm{e}$ & $7.18 \mathrm{c}-\mathrm{g}$ & $2.67 \mathrm{f}-\mathrm{h}$ & $2.56 \mathrm{a}-\mathrm{c}$ \\
\hline & TM524 & $53.33 \mathrm{e}-\mathrm{g}$ & $5.78 \mathrm{~b}-\mathrm{d}$ & $4.22 \mathrm{~g}$ & $32.33 \mathrm{ab}$ & $84.33 a-e$ & $85.24 \mathrm{a}-\mathrm{c}$ & $4.81 \mathrm{f}$ & $6.04 \mathrm{a}$ & $7.33 \mathrm{c}-\mathrm{g}$ & $4.00 \mathrm{~b}-\mathrm{d}$ & $2.79 \mathrm{ab}$ \\
\hline
\end{tabular}

Values with same letter(s) are statistically identical at 5\% level of probability 
Table 10. Mean performance for 11 traits of genotypes of cluster III \& IV

\begin{tabular}{|c|c|c|c|c|c|c|c|c|c|c|c|c|}
\hline Cluster & Genotype & DFPF & FPC (no.) & FRPC (no.) & FPP (no.) & $\mathrm{PH}(\mathrm{cm})$ & FW (g) & $\mathrm{FL}(\mathrm{cm})$ & $\mathrm{FD}(\mathrm{cm})$ & PT (mm) & LN & FYP $(\mathrm{Kg})$ \\
\hline \multirow{16}{*}{ III } & TM343 & $52.33 \mathrm{~g}$ & $8.33 a$ & $7.00 \mathrm{a}$ & $34.11 \mathrm{~b}-\mathrm{d}$ & $76.25 \mathrm{e}$ & $60.98 \mathrm{~cd}$ & $6.16 \mathrm{~b}$ & $4.73 b-d$ & 7.78a-d & $2.00 \mathrm{f}$ & $2.09 \mathrm{~b}-\mathrm{e}$ \\
\hline & TM369 & $68.67 \mathrm{~cd}$ & $7.33 \mathrm{ab}$ & $5.35 \mathrm{c}-\mathrm{e}$ & $32.17 \mathrm{~b}-\mathrm{e}$ & $91.73 b-d$ & $47.06 \mathrm{~d}$ & $4.15 \mathrm{f}$ & $4.90 \mathrm{~b}-\mathrm{d}$ & $6.50 \mathrm{~d}-\mathrm{f}$ & $3.33 \mathrm{~cd}$ & $1.51 \mathrm{de}$ \\
\hline & TM374 & $56.00 \mathrm{ef}$ & $5.67 \mathrm{c}-\mathrm{f}$ & $5.00 \mathrm{c}-\mathrm{f}$ & $20.22 \mathrm{ef}$ & $85.70 \mathrm{c}-\mathrm{e}$ & $119.37 \mathrm{a}$ & $5.64 \mathrm{~b}-\mathrm{d}$ & $6.07 \mathrm{a}$ & $7.50 \mathrm{a}-\mathrm{e}$ & $4.67 \mathrm{~b}$ & $2.40 \mathrm{~b}-\mathrm{d}$ \\
\hline & TM403 & $54.00 \mathrm{fg}$ & $7.00 \mathrm{a}-\mathrm{c}$ & $5.56 \mathrm{~cd}$ & $22.58 \mathrm{~d}-\mathrm{f}$ & $75.01 \mathrm{e}$ & $83.41 \mathrm{bc}$ & $5.86 \mathrm{bc}$ & $5.56 \mathrm{a}-\mathrm{c}$ & $8.46 a b$ & $2.58 \mathrm{~d}-\mathrm{f}$ & $1.88 \mathrm{c}-\mathrm{e}$ \\
\hline & TM404 & $56.00 \mathrm{ef}$ & 6.33b-e & $5.78 \mathrm{bc}$ & $21.33 \mathrm{~d}-\mathrm{f}$ & $75.18 \mathrm{e}$ & $61.39 \mathrm{~cd}$ & $5.20 \mathrm{~cd}$ & $5.07 \mathrm{~b}-\mathrm{d}$ & $8.00 \mathrm{a}-\mathrm{c}$ & $2.00 \mathrm{f}$ & $1.24 \mathrm{de}$ \\
\hline & TM417 & $67.00 \mathrm{~d}$ & $7.45 \mathrm{ab}$ & $6.75 \mathrm{ab}$ & $30.19 b-f$ & 79.50de & $54.26 \mathrm{~d}$ & $4.09 \mathrm{f}$ & $5.14 \mathrm{~b}-\mathrm{d}$ & $7.18 \mathrm{~b}-\mathrm{f}$ & $4.00 \mathrm{bc}$ & $1.59 \mathrm{de}$ \\
\hline & TM420 & $77.67 \mathrm{a}$ & $6.12 \mathrm{~b}-\mathrm{e}$ & $6.67 \mathrm{ab}$ & $33.75 \mathrm{~b}-\mathrm{e}$ & $75.50 \mathrm{e}$ & 63.43b-d & $4.00 \mathrm{f}$ & $5.48 \mathrm{a}-\mathrm{d}$ & $6.18 \mathrm{ef}$ & $5.67 \mathrm{a}$ & $2.15 \mathrm{~b}-\mathrm{e}$ \\
\hline & TM428 & $52.67 \mathrm{~g}$ & $5.22 \mathrm{ef}$ & $5.44 \mathrm{c}-\mathrm{e}$ & $25.33 \mathrm{c}-\mathrm{f}$ & $93.68 \mathrm{bc}$ & $58.50 \mathrm{~cd}$ & $5.00 \mathrm{de}$ & $5.03 \mathrm{~b}-\mathrm{d}$ & $6.33 \mathrm{ef}$ & $2.00 \mathrm{f}$ & $1.50 \mathrm{de}$ \\
\hline & TM462 & $69.33 \mathrm{~cd}$ & $7.34 \mathrm{ab}$ & $5.56 \mathrm{~cd}$ & $21.67 \mathrm{~d}-\mathrm{f}$ & $84.00 \mathrm{c}-\mathrm{e}$ & $61.72 \mathrm{~cd}$ & $4.32 \mathrm{ef}$ & $4.87 \mathrm{~b}-\mathrm{d}$ & $6.00 \mathrm{f}$ & $3.17 \mathrm{c}-\mathrm{d}$ & $1.23 \mathrm{de}$ \\
\hline & TM466 & $71.33 \mathrm{bc}$ & $5.67 \mathrm{c}-\mathrm{f}$ & $6.75 \mathrm{ab}$ & $40.78 \mathrm{ab}$ & $82.93 \mathrm{c}-\mathrm{e}$ & $71.56 \mathrm{~b}-\mathrm{d}$ & $7.03 \mathrm{a}$ & $4.90 \mathrm{~b}-\mathrm{d}$ & $7.89 \mathrm{a}-\mathrm{c}$ & $2.00 \mathrm{f}$ & $2.85 \mathrm{bc}$ \\
\hline & TM412 & $57.00 \mathrm{ef}$ & $5.56 \mathrm{~d}-\mathrm{f}$ & $6.67 \mathrm{ab}$ & $42.83 \mathrm{ab}$ & 79.67de & $71.15 b-d$ & $6.91 \mathrm{a}$ & $4.70 \mathrm{~cd}$ & $7.78 \mathrm{a}-\mathrm{d}$ & $2.44 \mathrm{ef}$ & $3.18 \mathrm{~b}$ \\
\hline & TM394 & $73.67 \mathrm{~b}$ & $6.22 \mathrm{~b}-\mathrm{e}$ & $4.44 \mathrm{ef}$ & $37.50 \mathrm{a}-\mathrm{c}$ & $89.67 b-d$ & $60.59 \mathrm{~cd}$ & $5.05 \mathrm{de}$ & $5.07 \mathrm{~b}-\mathrm{d}$ & $6.83 \mathrm{c}-\mathrm{f}$ & $3.33 \mathrm{~cd}$ & $2.29 \mathrm{~b}-\mathrm{d}$ \\
\hline & TM349 & $55.00 \mathrm{eg}$ & $4.67 \mathrm{f}$ & $4.22 \mathrm{f}$ & $17.33 \mathrm{f}$ & $105.67 \mathrm{a}$ & $56.01 \mathrm{~cd}$ & $5.37 \mathrm{~cd}$ & $4.67 \mathrm{~cd}$ & $7.50 \mathrm{a}-\mathrm{e}$ & $2.00 \mathrm{f}$ & $0.98 \mathrm{e}$ \\
\hline & TM514 & $69.00 \mathrm{~cd}$ & $6.67 \mathrm{~b}-\mathrm{d}$ & $5.44 \mathrm{c}-\mathrm{e}$ & $39.33 a b$ & $109.50 \mathrm{a}$ & $49.01 \mathrm{~d}$ & $5.53 \mathrm{~b}-\mathrm{d}$ & $4.60 \mathrm{~d}$ & $8.57 \mathrm{a}$ & $2.00 \mathrm{f}$ & $1.91 \mathrm{c}-\mathrm{e}$ \\
\hline & TM515 & $73.00 \mathrm{~b}$ & $7.11 \mathrm{ab}$ & $6.67 \mathrm{ab}$ & $39.44 a b$ & $98.22 \mathrm{ab}$ & $54.25 \mathrm{~d}$ & $5.87 \mathrm{bc}$ & $4.63 \mathrm{~d}$ & $8.44 \mathrm{ab}$ & $2.00 \mathrm{f}$ & $2.12 \mathrm{~b}-\mathrm{e}$ \\
\hline & TM508 & $57.67 \mathrm{e}$ & $6.41 \mathrm{~b}-\mathrm{e}$ & $4.67 \mathrm{~d}-\mathrm{f}$ & $48.82 \mathrm{a}$ & $86.72 \mathrm{~b}-\mathrm{e}$ & $89.79 b$ & $5.00 \mathrm{de}$ & $5.63 \mathrm{ab}$ & $7.96 \mathrm{a}-\mathrm{c}$ & $3.67 \mathrm{c}$ & $4.31 \mathrm{a}$ \\
\hline \multirow{13}{*}{ IV } & TM375 & $56.33 \mathrm{c}$ & $4.33 \mathrm{~d}$ & $5.35 \mathrm{bc}$ & $22.83 a-d$ & $93.49 \mathrm{bc}$ & $109.24 a$ & $5.75 \mathrm{~d}$ & $5.78 \mathrm{a}-\mathrm{d}$ & $8.17 \mathrm{~b}-\mathrm{d}$ & $4.00 \mathrm{ab}$ & $2.51 \mathrm{ab}$ \\
\hline & TM383 & $54.33 \mathrm{c}-\mathrm{e}$ & $5.11 \mathrm{~cd}$ & $4.78 \mathrm{bc}$ & $18.67 \mathrm{~b}-\mathrm{e}$ & $89.04 \mathrm{bc}$ & $98.55 \mathrm{a}$ & $5.32 \mathrm{~d}$ & $5.73 \mathrm{a}-\mathrm{d}$ & $7.33 c-e$ & $4.11 \mathrm{ab}$ & $1.80 \mathrm{~b}-\mathrm{d}$ \\
\hline & TM388 & $55.33 \mathrm{~cd}$ & $5.56 \mathrm{~cd}$ & $4.22 \mathrm{c}$ & $22.67 \mathrm{a}-\mathrm{d}$ & $105.09 \mathrm{a}$ & $108.99 \mathrm{a}$ & $5.53 \mathrm{~d}$ & $6.11 \mathrm{a}$ & $6.50 \mathrm{e}$ & $4.89 \mathrm{a}$ & $2.49 \mathrm{ab}$ \\
\hline & TM390 & $54.33 \mathrm{c}-\mathrm{e}$ & $5.33 \mathrm{~cd}$ & $4.78 b c$ & $23.92 \mathrm{a}-\mathrm{c}$ & 79.76de & $99.51 \mathrm{a}$ & $5.63 \mathrm{~d}$ & $5.68 \mathrm{a}-\mathrm{d}$ & $6.88 \mathrm{de}$ & $3.92 \mathrm{ab}$ & $2.35 \mathrm{a}-\mathrm{c}$ \\
\hline & TM392 & $55.33 \mathrm{~cd}$ & $5.67 \mathrm{c}$ & $4.67 \mathrm{bc}$ & $19.17 \mathrm{a}-\mathrm{e}$ & $86.36 \mathrm{~cd}$ & $97.96 \mathrm{a}$ & $5.38 \mathrm{~d}$ & $5.95 \mathrm{a}-\mathrm{c}$ & $6.83 \mathrm{de}$ & $4.17 \mathrm{ab}$ & $1.88 \mathrm{~b}-\mathrm{d}$ \\
\hline & TM393 & $53.67 \mathrm{c}-\mathrm{e}$ & $7.11 \mathrm{ab}$ & $5.00 \mathrm{bc}$ & $26.00 \mathrm{a}$ & $93.78 \mathrm{bc}$ & $96.78 \mathrm{a}$ & $6.78 \mathrm{bc}$ & $5.40 \mathrm{~cd}$ & $9.71 \mathrm{a}$ & $2.42 \mathrm{~d}$ & $2.53 \mathrm{ab}$ \\
\hline & TM407 & $52.33 \mathrm{e}$ & $5.67 \mathrm{c}$ & $4.33 \mathrm{c}$ & $17.50 \mathrm{c}-\mathrm{e}$ & $85.88 \mathrm{~cd}$ & $111.49 \mathrm{a}$ & $5.34 \mathrm{~d}$ & $6.14 \mathrm{a}$ & 7.19c-e & $4.83 \mathrm{a}$ & $1.94 \mathrm{a}-\mathrm{d}$ \\
\hline & TM408 & $52.67 \mathrm{de}$ & $5.78 \mathrm{c}$ & $4.67 b c$ & $15.75 \mathrm{de}$ & $93.32 \mathrm{bc}$ & $102.58 \mathrm{a}$ & $5.59 \mathrm{~d}$ & $5.79 \mathrm{a}-\mathrm{d}$ & $7.33 \mathrm{c}-\mathrm{e}$ & $4.40 \mathrm{ab}$ & $1.62 \mathrm{~cd}$ \\
\hline & TM410 & $66.00 \mathrm{a}$ & $7.33 \mathrm{a}$ & $5.96 \mathrm{ab}$ & $15.67 \mathrm{de}$ & $75.33 \mathrm{e}$ & $100.16 \mathrm{a}$ & $5.72 \mathrm{~d}$ & $5.80 \mathrm{a}-\mathrm{d}$ & $7.17 \mathrm{c}-\mathrm{e}$ & $3.50 \mathrm{bc}$ & $1.52 \mathrm{~d}$ \\
\hline & TM445 & $56.00 \mathrm{c}$ & $6.00 \mathrm{bc}$ & 7.11a & $14.00 \mathrm{e}$ & $92.50 \mathrm{bc}$ & $104.88 \mathrm{a}$ & 7.41a & $5.36 \mathrm{~d}$ & $8.40 \mathrm{a}-\mathrm{c}$ & $2.33 \mathrm{~d}$ & $1.48 \mathrm{~d}$ \\
\hline & TM497 & $56.33 \mathrm{c}$ & $6.44 \mathrm{a}-\mathrm{c}$ & $4.44 b c$ & $16.23 \mathrm{de}$ & $95.63 \mathrm{~b}$ & $90.67 \mathrm{a}$ & $5.69 \mathrm{~d}$ & $6.02 \mathrm{ab}$ & $8.50 \mathrm{a}-\mathrm{c}$ & $2.89 \mathrm{~cd}$ & $1.42 \mathrm{~d}$ \\
\hline & TM458 & 64.67ab & $6.11 \mathrm{a}-\mathrm{c}$ & $5.00 \mathrm{bc}$ & $25.44 a b$ & $86.26 \mathrm{~cd}$ & $108.70 \mathrm{a}$ & $7.23 \mathrm{ab}$ & $5.81 \mathrm{a}-\mathrm{d}$ & $9.37 \mathrm{ab}$ & $2.00 \mathrm{~d}$ & $2.75 \mathrm{a}$ \\
\hline & TM460 & $63.00 \mathrm{~b}$ & $5.56 \mathrm{~cd}$ & $5.35 \mathrm{bc}$ & $15.67 \mathrm{de}$ & $90.50 \mathrm{bc}$ & $102.34 \mathrm{a}$ & $6.53 \mathrm{c}$ & $5.50 \mathrm{~b}-\mathrm{d}$ & $8.50 \mathrm{a}-\mathrm{c}$ & $2.75 \mathrm{~cd}$ & $1.60 \mathrm{~cd}$ \\
\hline
\end{tabular}

Table 11. Parent selection from different clusters and their saline features

\begin{tabular}{cll}
\hline Cluster & \multicolumn{1}{c}{ Salient features } & \multicolumn{1}{c}{ Selected parent genotypes } \\
\hline I & Early flowering & TM409, TM386, TM528 as male \\
& $\begin{array}{l}\text { Tall plant } \\
\text { High fruit yield }\end{array}$ & \\
\hline II & Early flowering & \\
& Medium yielder & TM356, TM361, TM368, TM371, TM377, TM384, \\
& TM422, TM423 as female \\
\hline III & High flower and fruit per cluster & TM403, TM349 as male \\
& High fruits per plant & \\
\hline IV & Longer fruit & TM388, TM390, TM392,TM410 as Female \\
& Higher fruit weight & \\
\hline V & Highest fruit weight & TM382, TM419, TM360 as Female \\
& More fruit diameter & \\
& More pericarp thickness & \\
& More locule number per fruit & \\
\hline
\end{tabular}




\section{DISCUSSION}

The clustering pattern indicates that there was no association between geographical distribution of genotypes and genetic divergence. This result suggests that the factor(s) other than geographical separation is responsible for divergence. Singh et al. (2008) reported eight clustering in tomato, Sharma et al. (2006) studied with 60 genotypes of tomato and had 10 clusters and Prasanth (2003) had seven clusters for 67 tomato genotypes. The present genotypes were grouped into five clusters. Among the five clusters, cluster II was the biggest with 23 genotypes, followed by III and IV with 16 and 13 genotypes. This result supported to the Mahesh et al. (2006) results, they reported 11 genotypes in cluster II and Singh et al. (2008) reported 10 each in cluster I \& II.

The maximum inter cluster distance recorded between III and V clusters (8.34) indicate to obtain wide variability of the genotypes in these clusters. The moderate distance was between cluster I and II (5.27). It was apparent that the genotypes included cluster III was far diversed from cluster V and where genotypes belonging to V and IV were the least diversed followed by IV and II. Genotypes of cluster IIIIV, III-I and I-V were moderately diversed from each other. Therefore, the genotypes present in the clusters III and V or I and II could be utilized for successful hybridization progarmme. Inter cluster distance was observed maximum between cluster V and VI by Prasanth (2003). Considerable diversity between clusters was noticed by Dharmatti et al. (2001). Hybridization among the genotypes drawn from widely divergent clusters with high yield potential would likely to manifest maximum heterotic combinations as well as new recombination with desired traits. The intra cluster distance was maximum in cluster I (0.061) was invariably smaller than the lowest inter cluster divergence between clusters IV and V (2.83), thus authenticating the clustering pattern formed in this study. The maximum intra-cluster distance was reported by Prasanth (2003) in cluster IV. The intra cluster distances in all the five clusters were lower than the inter cluster distances and which indicated that genotypes within the same cluster were closely related.

First principal component axis contributed maximum $(25.31 \%)$ each towards total divergence followed by $2^{\text {nd }}$ PCA $(20.17 \%), 3^{\text {rd }}$ PCA $(15.01 \%), 4^{\text {th }}$ PCA $(13.10 \%), 5^{\text {th }}$ PCA $(8.55 \%)$ and $6^{\text {th }}$ PCA (6.99). These results were almost in accordance with the studies of Prasanth (2003).

Cluster I mainly an early flowering genotype e.g. it produced the lowest values for $50 \%$ flowering. They possessed the highest mean values for plant height and yield. This findings support to the result of Arun et al. (2003) for these traits. The genotypes under cluster II were also early flowering and higher yielder. Cluster III has late flowering and maximum flowers and fruits per cluster, fruits per plant and the lowest fruit weight, fruit length, fruit diameter, pericarp thickness, locule number per fruit. The genotypes belong to the cluster IV were oblong fruit and lowest number of fruits per cluster. The genotypes of the cluster $\mathrm{V}$ were shorter plant statue and the least 
number of fruits per plant. They also had larger fruit size along with most fruit diameter and pericarp thickness. In general, cluster $\mathrm{V}$ had maximum mean for most of the characters followed by cluster I including yield in the present study.

\section{Selection of parents}

From five clusters twenty parents were selected based on mean value within each cluster. From cluster I the genotypes TM409, TM386 and TM528 and from cluster III genotypes TM403 and TM349 were selected as male (tester) parent. Other hand, the female (line) parents were selected as TM356, TM361, TM368, TM371, TM377, TM384, TM422 and TM423 from cluster II; genotypes TM388, TM390, TM392 and TM410 from cluster IV; and genotypes TM382, TM419 and TM360 from cluster V for line $\mathrm{x}$ tester $(15 \mathrm{x} 5)$ cross.

\section{CONCLUSION}

Parent selection is an important step in any breeding program. In the present study, 20 genotypes out of 70 were selected using cluster analysis and mean performance. Cluster analysis developed uniform group and mean separation helped in selection parent from each uniform group. Genetic potentiality of the selected parents could be evaluated by combining ability and heterotic hybrid.

\section{REFRERRENCE}

Arun J., Kohil, U.K. and Joshi A. (2003). Genetic divergence for quantitative and qualitative traits in tomato (Lycopersicon esculentum Mill.). Indian Journal Agricultural Science, 73(2): 110-113.

Bai, Y. and Lindhot, P. (2007). Domestication and breeding of tomatoes: What have we gained and what can we gain in the future. Annals of Botany, 100(5): 1085-1094.

BBS, (2018). Yearbook of Agricultural Statistics, Bangladesh Bureau of Statistics. Statistics Division, Ministry of Planning, Dhaka, Bangladesh. pp. 111.

Dharmatti, P.R., Madalgeri B.B., Patil, R.Y., Mannikeri, I.M. and Girish, P. (2001). Combining ability studies in summer tomato. Karnataka Journal of Agricultural Science, 14(2): 417-422.

Mahalanobis, P.C. (1936). On the generalized distance in statistics. Proceedings of National Academy of Science (India), 2: 49-55.

Mahesh, D.K., Apte, V.B. and Jadhav, B.B. (2006). Studies on genetic divergence in tomato. Crop Research, 32(3): 401-402.

Meena, O.P. and Bahadur, V. (2015). Breeding potential of indeterminate tomato (Solanum lycopersicum L.) Accessions using $\mathrm{D}^{2}$ Analysis. SABRAO Journal of Breeding and Genetics, 47(1): 49-59.

Nalla, M.K., Rana, M.K., Singh, S.J., Sinha, A.K., Reddy, P.K. and Mohapatra, P.P.1. (2014). Assessment of genetic diversity through D2 analysis in tomato (Solanum lycopersicon. L). International Journal of Innovation and Applied Studies, 6(3): 431-438. 
Prasanth, S.J. (2003). Variability and divergence studies in tomato. M.Sc.(Agri.) Thesis, University of Agricultural Science Dharwad (India).

Rao, C.R. (1952). Advanced Statistical Methods in Biometrical Research, John Wiley and Sons, New York, pp. 36-38.

Reddy, B.R., Reddy, M.P., Reddy, D.S. and Begum, H. (2013). Correlation and path analysis studies for yield and quality traits in tomato (Solanum lycopersicum L.). IOSR Journal of Agriculture and Veterinary Science, 4(4): 56-59.

Sharma, H.R., Sharma, D. and Thakur, A.K. (2006). Studies on analysis of genetic divergence in tomato (Lycopersicon esculentum Mill.). Journal of Horticultural Science, 1(1): 5254.

Singh, A.K., Sharma, J.P, Kumar, S. and Chopra, S. (2008). Genetic divergence in tomato (Lycopersicon esculentum mill.). Journal of Research, SKUAST-J, 7(1): 1-8. 